Fecha de recepción: abril 2021 Fecha de aprobación: mayo 2021 Fecha publicación: junio 2021

\section{Desde la bio-imitación a la bio- extrapolación: Diseño Basado en Simbiogénesis como medio para la resolución creativa biológicamente inspirada de integración inter-especies}

Alejandro Durán-Vargas ${ }^{(1)}$ y

Daniela Rojas-Levy ${ }^{(2)}$

Resumen: El origen de las ideas depende en muchos casos de la capacidad analógica que asume el diseñador para extrapolar las cualidades desde la fuente de inspiración. Cuando esta inspiración proviene desde lo natural, la reconocemos como Diseño Biológicamente Inspirado.

En el presente artículo se presenta un resumen de estas aproximaciones, junto al potencial del Diseño Basado en Simbiogénesis como modelo que además de reconocer el valor de la analogía biológica, incorpora dinámicas evolutivas asociadas al origen de las especies por medio de la cooperación y la integración por medio de la simbiosis.

Palabras clave: Diseño Biológicamente Inspirado (BID) - Diseño Basado en Simbiogénesis - Problem-driven BID - Solution-driven BID - simbionte.

[Resúmenes en inglés y portugués en la página 115]

(1) Licenciado en Diseño, Académico e Investigador de la Facultad de Arquitectura, Diseño y Estudios Urbanos de la Pontificia Universidad Católica de Chile; Doctorando en Ciencias Biológicas, mención Ecología, Pontificia Universidad Católica de Chile; Director Lowtech Design Group UC; Director ECCCO, Plataforma de Divulgación y Educación Científica; y Director Alterno SEISMO: Instrumento de diagnóstico digital para el catastro asistido de daños estructurales durante la post emergencia sísmica.aaduran@uc.cl

(2) Diseñadora de la Pontificia Universidad Católica de Chile; integrante de LowTech Design Group; Coordinadora de Diseño en ECCCO, Plataforma de Divulgación y Educación Científica; y Diseñadora UX/UI de SEISMO: Instrumento de diagnóstico digital para el catastro asistido de daños estructurales durante la post emergencia sísmica. 


\section{Introducción}

El origen de las ideas, a diferencia de "El Origen de las especies", obra maestra del naturalista inglés Charles Darwin, no posee un andamiaje evolutivo tan claro. No tenemos un ancestro común cuando hablamos de las creaciones humanas. Tampoco podemos declarar que las ideas mejor adaptadas puedan sobrevivir en el tiempo.

Cuando hablamos de ¿cómo surgen las ideas?, nos enfrentamos al desafío de entender procesos creativos, que en muchos casos, parecen emerger como actos espontáneos, mutaciones en el orden natural del proceso acumulativo de la cultura material que cambia la dirección de lo conocido y nos enfrenta a nuevos paradigmas.

La búsqueda de fuentes de inspiración, hace uso de nuestra capacidad de extrapolación para aprovechar las características de una entidad semejante y adaptar estas cualidades en ámbitos distintos. En palabras de Margaret Boden en su libro What is creativity? "Analogy seems to have a share in all discoveries, but in some it has the lion's share".

$\mathrm{Al}$ analizar el uso de analogías en los procesos creativos, específicamente en el diseño de nuevas soluciones, la distancia o frecuencia de la analogía, entendida como la cercanía de la fuente de inspiración al ámbito de solución, es uno de los factores claves a reconocer. Al momento de revisar la forma en que estas referencias son traducidas, nuestra aproximación puede enfocarse desde una perspectiva que se basa en la imitación del objeto como resultado que resuelve; o desde una inspiración que devela el procedimiento por el cuál, el objeto inspirador se constituyó y logra resolver el problema que esperamos solucionar. Esta gradiente que fluctúa desde la copia, a la imitación y luego a la extrapolación, nos lleva, sin importar el grado de distancia correlacional, a traspasar los dominios propios y ampliar el acotado repertorio de soluciones con los que un campo disciplinar dispone. Es un desafío transdisciplinar que nos interpela a traducir juegos de lenguaje (Cordua, 1996), comprender los mecanismos intrínsecos de lo observado y avanzar hacia una dinámica de apertura e integración proyectual.

Una persona entrenada en resolución y creación de propuestas de solución será capaz de conectar fuentes de inspiración distantes, reconociendo de forma abstracta los elementos a extrapolar. En el caso del Diseño, la ideación analógica ha sido usada como mecanismo para innovar, y cuando la inspiración surge desde lo natural, podemos denominarlo BID por su sigla en inglés, Diseño Biológicamente Inspirado. Vandevenne lo describe como la disciplina donde se toma la inspiración desde la naturaleza para resolver los problemas que enfrentan los humanos. A lo largo de su evolución se han generado múltiples aproximaciones metodológicas para la extrapolación de estas inspiraciones naturales en soluciones de diseño. BID se puede abordar utilizando diferentes paradigmas, a saber, BID impulsado por problemas [problem-driven BID], BID impulsado por soluciones [solution-driven BID], biomímesis y bio replicación (Lenau, Metze \& Hesselberg, 2018). Siendo el problem-driven BID y el solution-driven BID aquellas con mayor adherencia y desarrollo.

Problem-driven BID se presenta como una progresión de pasos que comienzan desde la definición del problema, su reformulación, búsqueda de soluciones biológicas, para terminar con la extracción del principio y su aplicación (Helms, Vattam and Goel, 2009). 
Por otra parte, solution-driven BID es menos sistemática y ocurre típicamente como resultado de descubrimientos fortuitos de fascinantes procesos o mecanismos biológicos (Lenau et al, 2018).

En el caso del problem-driven BID la dificultad está en conocer el amplio repertorio de adaptaciones naturales existentes, no solo por la distancia disciplinar, sino además, por las diferencias lingüísticas. Dado que los objetos, las relaciones y los procesos son muy diferentes, los biólogos y diseñadores, ingenieros y arquitectos suelen hablar un idioma muy distinto, lo que crea desafíos de comunicación (Helms et al, 2009). Por otro lado, las solution-driven BID presentan la dificultad de la amplitud de posibles aplicaciones y el desafío de generar procedimientos que favorezcan su implementación.

En este sentido, la metodología de Diseño Basado en Simbiogénesis (Durán and O’Ryan, 2019) propone una estrategia que si bien comienza desde una aproximación problem-driven BID, con algunas adaptaciones, puede permitir la generación de un modelo enriquecido para las solution-driven BID. Diseño Basado en Simbiogénesis propone una estrategia de identificación de beneficios; observación divergente; y adaptación cooperativa de entidades complementarias, siendo una metodología orientada en los procesos de diseño y generación de nuevos conceptos de solución basado en la cooperación e integración de organismos (Durán and O’Ryan, 2019). Esta aproximación no solo permite contar con un procedimiento de diseño que surge desde la inspiración biológica, sino además, aprovechar una metodología que se basa en la búsqueda de analogías complementarias, brindando mayor robustez a las soluciones producidas.

En este artículo se presenta una revisión general del Diseño Biológicamente Inspirado, un resumen de las fases del modelo de Diseño Basado en Simbiogénesis junto a consideraciones para su adaptación en una aproximación bidireccional. Asimismo se describe el proceso de diseño de un caso aplicado. El diseño de un simbionte originado desde una perspectiva solution-driven junto a la integración del modelo de cooperación e integración propuesto por el Diseño Basado en Simbiogénesis. Shelley, Soft-robot acuático mitad sintético mitad orgánico capaz de oxigenar y filtrar el medio (Rojas and Durán, 2017).

Por último, se analiza el potencial de esta aproximación simbiótica de Diseño Biológicamente Inspirado como un posible camino hacia un modelo bidireccional de diseño analógico que cruza los dominios disciplinares tanto desde la extrapolación de la adaptación como de la inspiración en los mecanismos evolutivos de cooperación e integración inter-especies.

\section{Aproximaciones proyectuales Bio Inspiradas}

El Diseño biológicamente inspirado es un campo de desarrollo que atrae gran interés y que ha acompañado a la disciplina del Diseño desde su comienzo en múltiples facetas (Fayemi, Maranzana, Aoussat and Bersano, 2014). Cuando se habla de Diseño inspirado en la naturaleza, conceptos como biomímesis, bio replicación e incluso biónica se sacan a colación, muchas veces utilizándose como intercambiables cuando en realidad no se re- 
fieren a lo mismo. La disciplina se encuentra llena de conceptos que se superponen y que dificultan el entendimiento y delimitación del campo.

El concepto más usado es Bio Inspired Design o BID, "que a menudo se utiliza como sinónimo de biomímesis, pero también tiende a centrarse más en el proceso de Diseño" (Lenau et al, 2018). Biomímesis, por su parte, hace referencia a una cooperación entre disciplinas como la Biología, y la tecnología o la innovación para la resolución de problemas prácticos (ISO 18458, 2015). El término "biomimicry", considera la sustentabilidad como parte integral del proceso. En palabras del Biomimicry Institute:

Es una práctica que aprende e imita las estrategias utilizadas por las especies que habitan en la actualidad. El objetivo es crear productos, procesos y políticas -nuevas formas de vida- que resuelvan nuestros mayores desafíos de diseño de manera sostenible y solidaria con toda la vida en la tierra.

La biónica, por otro lado, busca utilizar dispositivos sintéticos para mejorar o incluso reemplazar alguna función biológica en particular. Este es el caso de las prótesis robóticas o los marcapasos. En contraste, la bio replicación es una copia "legítima" de alguna estructura, órgano o mecanismo natural. Una de sus funciones, guarda relación con poder entender en profundidad al menos una funcionalidad (Lenau et al, 2018), viéndola aplicada en la réplica.

Por último, la bio fabricación, también conocida como Growing Design (Camere and Karana, 2018), consta del proceso de producción de materiales y artefactos mediante el cultivo de organismos vivos y células (Mironov, 2009; Fujii, Yoshida \& Sugimura, 2016). A pesar de que este último campo puede considerarse parte de una categoría distinta, ya que no se inspira estrictamente en la naturaleza sino que utiliza organismos vivos para sus fines, un creciente número de profesionales trabajando en esta disciplina pretenden no sólo abordar las materias primas sino también inspirarse en cómo se forman las estructuras en la naturaleza para el desarrollo de biomateriales.

El siguiente diagrama, propuesto por Lenau, et al, (2018), presenta estas perspectivas en interrelación.

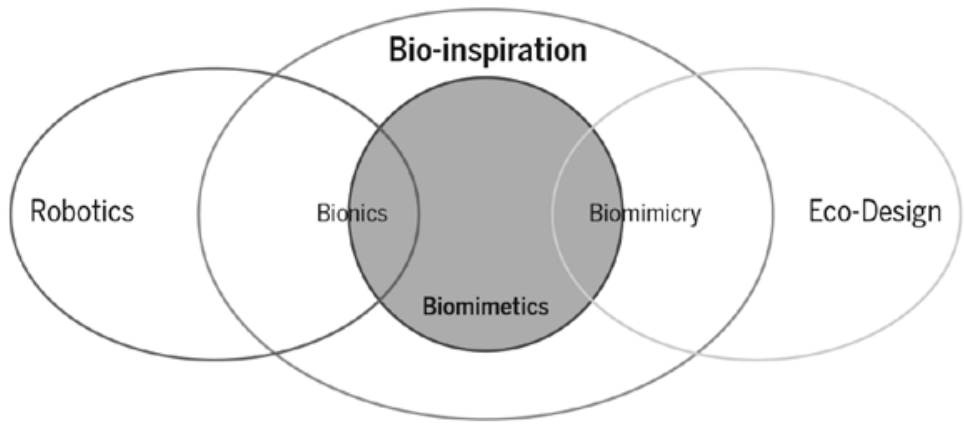


La biomímesis se ha consolidado como una de las principales aproximaciones disciplinares referidas al Diseño Biológicamente Inspirado. Esta perspectiva ha aportado con inspiración desde el reconocimiento de cualidades presentes en organismos biológicos para su traducción en el diseño de soluciones sintéticas. El estándar ISO la define como:

La cooperación interdisciplinaria de la biología y la tecnología u otros campos de innovación con el objetivo de resolver problemas prácticos a través del análisis de la función de los sistemas biológicos, su abstracción en modelos y la transferencia y aplicación de estos modelos a la solución (ISO 18458, 2015).

El Diseño Bio Inspirado es, por cierto, un proceso creativo donde se utiliza lo orgánico como guía para generar nuevas aproximaciones, productos y servicios. Para esto se deben generar conexiones entre prestaciones naturales y artificiales construyendo un paralelismo que permita adaptar una función biológica a un contexto distinto, seguramente a realizar con otro tipo de materiales y tecnología. Para este fin, se han propuesto metodologías que buscan facilitar y clarificar el proceso de desarrollo de BID a un público más amplio y diverso.

En términos generales se habla de dos grandes paradigmas que dominan las metodologías BID: BID impulsado por problemas [problem-driven BID] y BID impulsado por soluciones [solution-driven BID]. Ambas se diferencian principalmente en el punto de partida, el primero comenzando por el problema a resolver mientras que el último comienza estrictamente desde la inspiración biológica.

\subsection{Metodologías Problem-driven BID}

Las metodologías problem-driven son aquellas en que el punto de partida está radicado en el problema. Es decir, el insumo inicial para el proceso es un problema para el cual se busca una solución inspirada en la naturaleza. También se les conoce como metodologías top-down y la mayoría de las metodologías BID existentes actualmente pertenecen a esta categoría . Por esto están bastante desarrolladas y descritas (Shu et al, 2011).

Un reconocido caso de diseño bioinspirado desde esta aproximación top-down son los Shinkansen o Japanese Bullet Trains. Estos medios de transporte urbano pueden alcanzar velocidades de más de $300 \mathrm{~km} / \mathrm{hr}$ y se consideran los ferrocarriles más rápidos del mundo. El diseño que poseen hoy en día surge de un problema acústico que solían tener: los cambios bruscos en la presión del aire, sumados a las altas velocidades a las que se desplazan, generaban un sonido tronador cada vez que la cabina frontal emergía de algún túnel (Fayemi et al, 2014). Este ruido, particularmente potente, perturbaba considerablemente a los residentes de las viviendas aledañas. El rediseño de este tren estuvo a cargo de Eiji Nakatsu quien en su momento era el Director of the Technical Development and Test Operation Department of JR-West. Nakatsu se inspiró básicamente en 3 organismos que se encuentran en la naturaleza para la mejora del Shinkansen, pero la principal referencia vino del martín pescador, un ave que evolucionó para poder sumergirse en el agua a cazar sin generar ruido alguno. 
El problema fundamental es el mismo en ambos mundos, hacer la transición de un ambiente de baja presión que es aire para el martín pescador, a un ambiente de alta presión que es agua para el martín pescador (Fayemi et al, 2014).

Los pasos o etapas que se desarrollan, en general, en las metodologías problem-driven se ven reflejadas en la metodología BID de Georgia Tech:

1. Se demarca el problema que se quiere resolver y se descompone al máximo en requerimientos.

2. Se define el problema en términos biológicos más amplios, en función de los requerimientos determinados en el paso anterior.

3. Búsqueda de funciones biológicas que puedan abordar los requerimientos. Es fundamental buscar de manera equilibrada: generar una búsqueda no muy restringida ni muy abierta.

4. Elección de uno o varios fenómenos naturales para extrapolar y entender toda su complejidad.

5. Se extraen los principios fundamentales que forman parte del fenómeno natural.

6. Aplicación del concepto elegido en el nuevo contexto para solucionar el problema.

En este último paso, cabe mencionar lo que plantean Helms, Vattam \& Goel: "Los diseñadores creaban con frecuencia soluciones compuestas seleccionando lo "mejor de" múltiples fuentes para satisfacer las demandas de la competencia, que clasificamos como analogías compuestas".

Otros ejemplos de este tipo de metodologías son la metodología BID ISO, el Design Spiral del Biomimicry Institute, la metodología BID de Paris Tech y el Diseño Basado en Simbiogénesis (Durán and O’Ryan, 2019), que siguen la misma lógica pero difieren en los pasos individuales a seguir.

\subsection{Metodologías Solution-driven BID}

Los procesos de BID, sin embargo, puede tomar una aproximación diferente. En vez de comenzar por el problema a resolver se puede comenzar desde un fenómeno natural fascinante para luego buscar en qué disciplina/reino aplicarlo. Para este paradigma, conocido como solution-driven BID, existe un número mucho menor de metodologías propuestas y se entiende que en general es un proceso más intuitivo que determinado por pasos secuenciales. En palabras de Fayemi, Maranzana, Aoussat \& Bersano:

El método basado en soluciones asume un sistema biológico que realiza una función que el ingeniero quiere emular como punto de partida. El proceso se centra en abstraer el sistema biológico para que el diseñador pueda utilizar el modelo funcional para inspirar un concepto de diseño de ingeniería.

Pareciera ser más complejo buscar una aplicación aleatoria a un fenómeno natural que un fenómeno aleatorio a un problema específico. Pero en la práctica la variedad de problemas 
posibles no es necesariamente mayor que el número de fenómenos naturales que existen, por lo que quizás el número de metodologías problem-driven tenga que ver meramente con una mayor similitud de esta aproximación con nuestros procesos naturales para resolver problemas.

Sin embargo, existen variados productos que han utilizado esta aproximación para el diseño de sus soluciones: "Se han fabricado materiales funcionales avanzados, como superficies superhidrófobas, basados en estructuras diseñadas por criaturas vivientes, como la hoja de loto prístina" (Risbuda \& Bartl, 2013). Basándose en las propiedades de autolimpieza que poseen estas estructura vegetales, llamada superhidrofobicidad, existen variadas aplicaciones que se aprovechan de estas características para generar pinturas y cubiertas tejadas antimanchas, revestimientos para textiles entre otros.

Esta aproximación bottom-up posee una serie de pasos generales para su implementación:

1. Identificación de la solución biológica.

2. Definir o describir la solución elegida: profundización del fenómeno en cuestión (partes, mecanismos y funcionamiento).

3. Extracción de principios.

4. Replantear la solución.

5. Búsqueda del problema a solucionar: exploración de múltiples problemas para los cuales esta solución/mecanismo puede ser una buena aproximación.

6. Definición del problema: especificar el problema que queremos solucionar en términos prácticos.

7. Aplicación de principios: Aplicar los principios derivados de la naturaleza para solucionar problemas prácticos humanos y evaluar la pertinencia y efectividad.

\section{Origen de las especies como fuente de inspiración en procesos de innovación}

Gran parte de estas aproximaciones proyectuales basan el surgimiento de un nuevo producto en la abstracción de adaptaciones biológicas hacia una respuesta sintética. Toman la forma en que lo natural respondió ante una presión adaptativa del entorno y lo traducen a una versión material.

Cuando el motivo de estas analogías es la creación de nuevas respuestas creativas, basarnos solamente en como se han adaptado las especies y no en cómo se han generado las nuevas especies parece una omisión importante.

En este sentido, las aproximaciones biomiméticas permiten innovar en el origen de soluciones por medio del uso analógico de una adaptación natural, y si bien, el número de posibles adaptaciones desde las cuáles buscar inspiración parece inagotable, esta forma de analogía no incluye un aspecto fundamental del origen de las especies: para autores como Lynn Margulis y Dorion Sagan, la cooperación simbiótica de largo plazo entre organismos complementarios es lo que condujo al origen de nuevas especies por medio de la simbiogénesis. Para Margulis, la simbiosis es crucial para la comprensión de la diversidad 
evolutiva. La novedad basada en la cooperación, la integración interespecífica incremental es el punto de entrada para entender la aparición de nuevos organismos.

La simbiogénesis es un proceso que requiere de la integración basada en cooperación de al menos dos organismos con denominaciones diferentes para el origen de un nuevo organismo (Margulis, \& Sagan, 2008).

Si la asociatividad e integración entre organismos promueve el surgimiento de nuevas especies, surge la interrogante sobre qué ocurre cuando los sistemas que interactúan y cooperan no son completamente biológicos. ¿Es posible extrapolar estas dinámicas evolutivas en el diseño de soluciones sintéticas? (Durán, 2020).

Si bien la simbiosis en espacios naturales requiere de condiciones ambientales específicas que posibilitan el encuentro y favorezcan la cooperación, en el caso de una simbiosis asistida, promover la fusión de dos o más entidades complementarias dependerá de las cualidades específicas que la diseñadora o diseñador desee integrar. De esta manera, replicar esta lógica de cooperación dependerá de los atributos independientes de los organismos individuales, que, en un ecosistema específico, serán beneficiosos mutuamente.

Si la biomímesis nos ha servido como medio de inspiración desde el mundo natural, la simbiogénesis nos interpela a expandir esta bio-inspiración hacia una observación divergente de múltiples organismos, que, de forma integrada y cooperativa, pueden guiar los procesos de ideación (Durán, 2020).

Diseño basado en Simbiogénesis es una metodología basada en los procesos biológicos de cooperación e integración como medio de innovación en Diseño.

\section{Diseño basado en simbiogénesis}

El Diseño Basado en Simbiogénesis se propone estructurar: una estrategia de identificación de prestaciones; observación divergente; y adaptación cooperativa de entidades complementarias, siendo una metodología orientada en los procesos de ideación y generación de nuevos conceptos de diseño (Durán, 2020).

Las fases que componen esta metodología (ver Figura 2) comienzan desde [1] la deconstrucción del fenómeno que se está desarrollando para lograr reconocer las prestaciones a resolver; [2] una búsqueda del estado del arte con el propósito de entender el ámbito investigado desde la caracterización de los antecedentes existentes. Categorizar los productos y sistemas con que previamente se ha resuelto el problema permite reconocer las tecnologías y aproximaciones resolutivas, y con ello, establecer métricas comparativas que nutrirán la siguiente fase. La próxima etapa propone [3] la generación de parámetros de evaluación. Una rúbrica comparativa para contrastar fusiones futuras con las soluciones existentes. Tras la caracterización de los antecedentes, se tabula la información según campos cuantificables. Si bien esta etapa está supeditada a una evaluación temprana del desempeño de las soluciones generadas, en caso de ser posible, contar con informantes claves permite incluir una valoración perceptual de preferencias, lo que enriquecerá la comparación, incluyendo una perspectiva simbólica al proceso de ideación. 


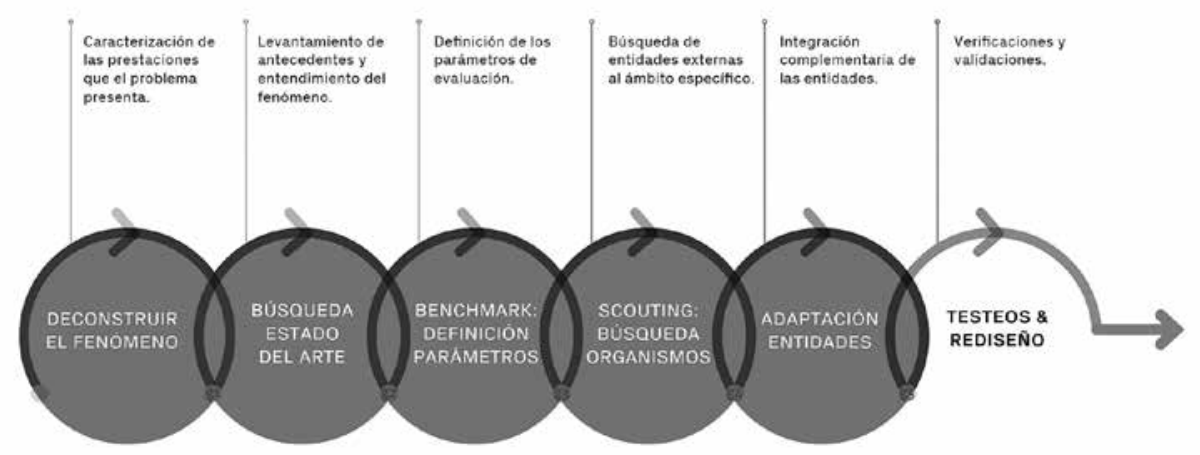

Figura 2.

Una de las fases más relevantes para el éxito de la propuesta es el [4] Scouting o búsqueda de entidades complementarias. Para obtener resultados diversos es importante realizar una búsqueda transversal de especies que cumplan con las prestaciones definidas en la fase inicial de la investigación. Al igual como organismos de diferentes linajes pueden convivir en simbiosis; Productos, servicios, sistemas o fenómenos conocidos y distanciados tipológicamente pueden nutrir una solución basada en cooperación e integración. Para esto se propone una búsqueda ampliada de referentes utilizando múltiples fuentes de información: Keywords utilizados en las publicaciones referidas a las prestaciones iniciales, Clasificación Internacional de Patentes (CIP) para propiedades intelectuales y derechos de autor, sumado a una búsqueda divergente de entidades que respondan a las prestaciones iniciales. La pesquisa debe asumir que se encontrarán las referencias en organismos complejos que no solo responderán a la prestación específica. De esa manera, el organismo a fusionar no será la totalidad de la entidad sino aquel subelemento portador del beneficio esperado.

La búsqueda de estas entidades complementarias no debe estar supeditada al mismo ámbito de acción original, en muchos casos, distintas categorías podrán brindar integraciones inesperadas y con alto potencial de efectividad.

La fase siguiente corresponde a la [5] adaptación y fusión de los organismos seleccionados desde la búsqueda de especies. Esta unión plantea un desafío de abstracción, composición y configuración, propios de la disciplina del diseño. Durante esta etapa, las partes y funciones de los referentes independientes son integrados en una nueva entidad. La unión de las entidades precursoras requiere de un proceso de resolución y prototipado iterativo que aproveche las ventajas individuales en pos de lograr un beneficio común. El grado de integración dependerá de las posibilidades complementarias de sus componentes. No es necesario evidenciar los precursores de una nueva especie, solo permitir el complemento. La última fase del modelo propone [6] la realización de evaluaciones comparativas de los resultados generados. Si bien existen múltiples aproximaciones metodológicas, ins- 
trumentos de validación y estudios de usabilidad (Dumas \& Redish, 1999), para esta propuesta metodológica se simplifica el estudio a un análisis comparativo según los parámetros expuestos en la fase 3. De esta manera se compara el producto de la fusión con los antecedentes mejor calificados en la búsqueda del estado del arte.

\section{Shelley: simbionte orgánico-sintético como caso de estudio al Diseño Basado en Simbiogénesis}

Shelley (ver Figura 3) es un Soft-robot sintético-natural acuático, energéticamente sustentable; oxigenador por movimiento y purificador por organismos vegetales. Es el resultado de un proyecto académico desarrollado el año 2017 por la diseñadora Daniela Rojas bajo la tutela de quien escribe este artículo. Su propósito era poner a prueba el modelo metodológico basado en la fusión de entidades complementarias para la resolución de un problema contingente. El proceso de desarrollo hizo uso de una versión recursiva de la metodología de Diseño Basado en Simbiogénesis; primero se trabajó desde una perspectiva solution-driven buscando tecnologías y fenómenos de interés, extrayendo los beneficios en abstracto de cada elemento analizado para determinar un ámbito de investigación pertinente (cultivo autónomos acuapónicos), y desde esta revisión ampliada, se comenzó siguiendo los pasos de la metodología descrita anteriormente.

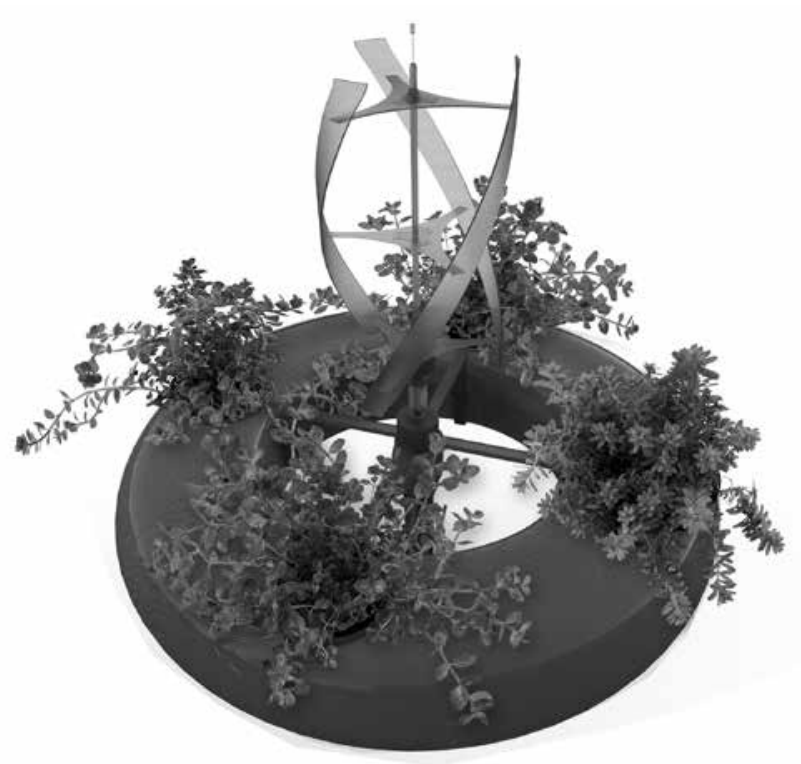

Figura 3. 
A continuación, se presenta parte del proceso y una descripción de la fisiología de este nuevo organismo compuesto por soft robotics (movimiento), biopelículas de nanocelulosa (capilaridad y empuje del agua) y organismos vegetales (filtración) que se mantiene flotando sobre el medio acuoso, oxigenando y purificando el agua que la rodea.

En este proceso de hibridación proyectual se tuvo como objetivo principal, proveer al nuevo organismo de la capacidad para oxigenar y purificar el agua que lo rodea de forma autónoma. Es así como las decisiones "etológicas" y morfológicas responden a la predicción de su posible comportamiento y desempeño en entornos de implementación, asumiendo que existen variables que deben ser contrastadas con su evolución en el tiempo (e.g. anidación de aves, crecimiento de organismos en su estructura entre otros), y bajo condiciones extremas de ecosistemas.

En este sentido, la forma de Shelley es el resultado acumulativo de sus predecesores. Su fisiología consta de [1] un "sistema cardíaco" que provee energía desde un capturador eólico; [2] un cuerpo toroidal ahuecado que le brinda flotabilidad y soporte a las especies vegetales que lo componen; [3] un sistema propulsor que transforma la energía eólica en movimiento lineal; [4] extremidades que generan el movimiento constante de las [5] membranas que nutren e irrigan las plantas, además de oxigenar el agua desde su agitación.

\subsection{Conversión de energía}

El sistema de conversión energética es el corazón de Shelley. El corazón es el órgano principal del sistema circulatorio; es aquel que irriga todos los músculos del cuerpo y permite la oxigenación de cada una de las células. Se trata de una bomba que moviliza los nutrientes en nuestro sistema. De la misma forma, Shelley cuenta con una bomba de energía que da potencia a todo el organismo: una hélice que al girar activa el sistema motor, el que mueve las extremidades poniendo en marcha las extremidades y sus membranas. Uno de los principales problemas durante el proceso de diseño de Shelley, fue el tipo de conversor de energía a utilizar. Se analizaron capturas fotovoltaicas, cinéticas y por cambio de presión ambiental, culminado con la decisión de un sistema eólico. En la elección del tipo de hélice se reconoció los problemas que su tamaño y disposición podría generar: enganche y choque con las plantas del sistema. El sistema elegido aprovecha la versatilidad y robustez de las hélices verticales (Figura 4), sistemas que giran sobre el eje vertical, y de los cuáles podemos reconocer dos tipos: Savonius y Darrieus. Para fines de este proyecto se escogió Darrieus gracias a sus ventajas comparativas que guardan relación con que no necesitan de una orientación específica en cuanto al viento para poder girar, resisten mejor a los vientos fuertes y no requieren de una torre de estructura muy resistente, ya que pueden funcionar incluso cerca del suelo, a diferencia de las llamadas hélices horizontales. 


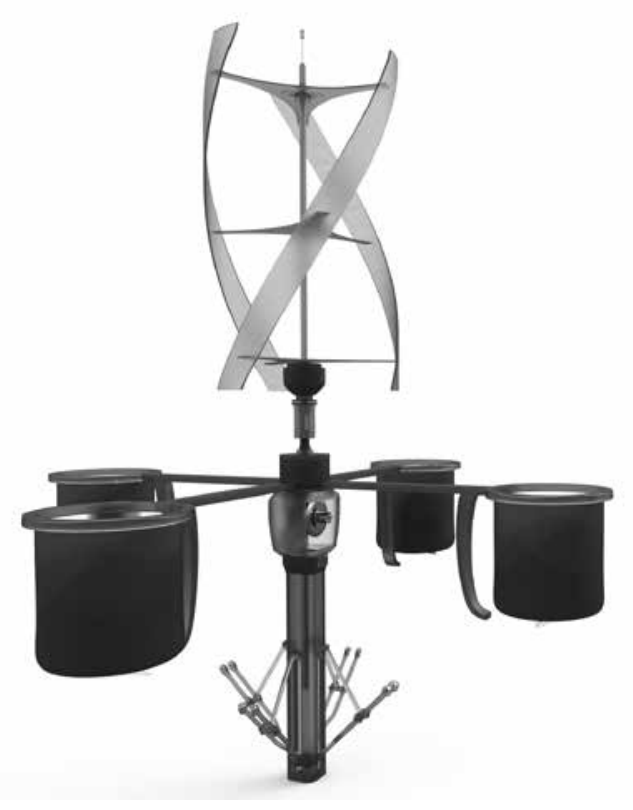

Figura 4 .

\subsection{Cuerpo toroidal}

No existe en la naturaleza un único tipo de fisionomía flotante. Esto se explica debido a que no todos los organismos responden a las mismas presiones adaptativas, tampoco ocupan los mismos nichos ecológicos. Es claro que tanto la forma y características de los cuerpos de los organismos han evolucionado para responder a las necesidades que cada especie posee en interacción con su entorno.

El cuerpo de Shelley, ha sido diseñado para poder flotar sobre el agua y soportar la carga de organismos vegetales que vivirán sobre su estructura y el sistema eólico de captura de movimiento. Debido a esto, se propone una estructura toroidal como soporte de los organismo vegetales, esta estructura contiene una gran masa de aire en su interior y se nivela tanto la parte superior como la inferior para generar un mejor equilibrio sobre el agua y favorecer el espacio que se utiliza para la ubicación de las plantas.

Como se puede apreciar en la Figura 5, existen espacios para cada una de las plantas y ranuras que permiten que membranas porosas puedan irrigar el sustrato por medio de la capilaridad de estos organelos. Estas membranas son "tentáculos" de nanocelulosa, biofilm que no solo permite el riego, además son la superficie de las extremidades del organismo. Se ingresa el biofilm por el espacio ya descrito y toda la estructura se conecta al sistema de conversión de energía por medio de brazos de biopolímero. Su unión ayuda a la triangulación y correcta distribución del peso que recibirán. 


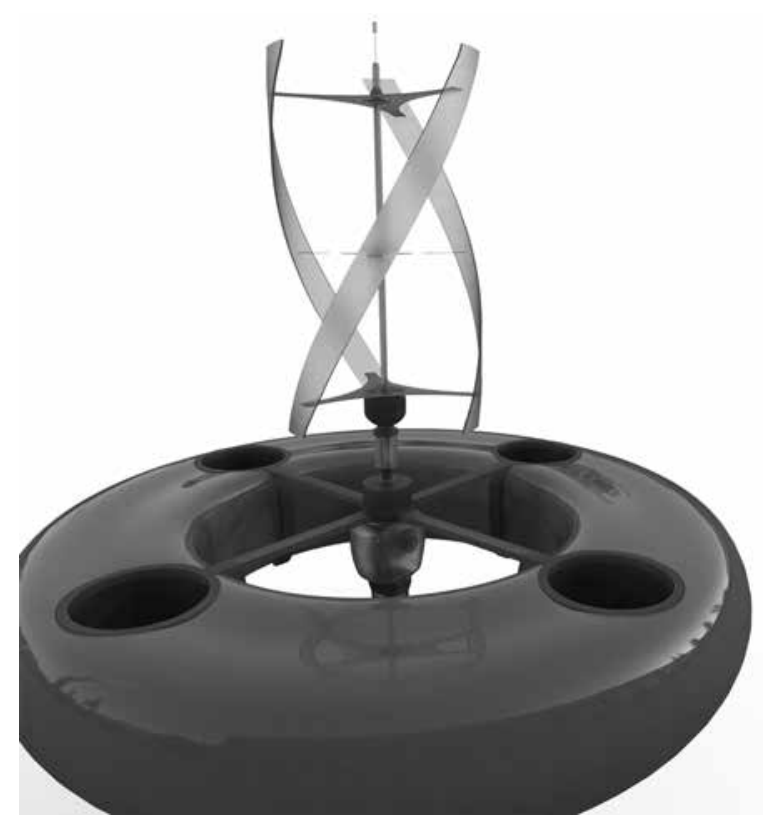

Figura 5.

\subsection{Extremidades}

Las extremidades son aquellas partes articuladas del organismo que cumplen funciones de locomoción. En el caso de Shelley, sus efectores no están diseñados para el desplazamiento del organismo, sino para movilizar el agua a su alrededor, oxigenando el medio acuoso y facilitando la distribución homogénea de los nutrientes existentes en el entorno. Permiten la oscilación de la membrana de nanocelulosa, y por ende, el empuje hidrodinámico del agua que rodea al organismo. Utiliza un pistón que, activado por el movimiento giratorio de la hélice, se encarga de subir y bajar. Esta pieza tiene una longitud de $155 \mathrm{~mm}$ y se conecta desde el mecanismo de conversión del movimiento de la hélice hacia los pivotes que movilizan el biofilm de nanocelulosa en la sección inferior del mecanismo (ver Figura 6). En esta última fracción del sistema, el pistón transita su camino por dentro de una pieza que funciona como "guía" de su recorrido. Ésta se trata de un paralelepípedo que se engancha al sistema motor gracias a un encaje en su extremo superior. Sumado a esto posee 4 calados, uno en cada una de sus caras, en la mitad inferior de su altura. Éstos permiten la conexión del pistón a las aletas de nanocelulosa. 



Figura 6.

\subsection{Membranas de irrigación y distribución}

Las membranas de irrigación están conformadas por biopelículas, superficies que generalmente recubren a un órgano, organismo o comunidad de organismos, permitiendo la interacción de éste con su ambiente dependiendo de las características del tejido mismo. En este caso, la membrana a utilizar, es un biofilm de nanocelulosa generada por la bacteria Acetobacter Aceti. Esta biopelícula es producida por un consorcio de microorganismos, quienes convierten nutrientes y azúcares presentes en su medio de cultivo en una red de exopolisacáridos y filamentos de nanocelulosa.

Como se mencionó anteriormente, las láminas de biopelícula se ingresan por la parte inferior del toroide y se fijan al sustrato que contendrá el material vegetal. La membrana posee dimensiones que fluctúan entre los 120 y $140 \mathrm{~mm}$ de largo y 35 a $45 \mathrm{~mm}$ de ancho. Estas membranas se conectan en su zona media con las extremidades, lo que permite que el movimiento rotatorio de la hélice se convierta en un desplazamiento horizontal, generando el empuje del medio acuoso.

\subsection{Organismos vegetales}

En relación al tipo de organismo vegetal a utilizar, debe cumplir ciertos requisitos: sistemas radiculares poco invasivos, poseer un crecimiento idealmente rastrero y consumir 
gran cantidad de agua. Bajo estas condiciones se decide utilizar Nasturtium Officinale, comúnmente conocido como berro. Este organismo posee, en primer lugar, un crecimiento rastrero; es por esto que es común encontrarlas a las orillas de ríos y fuentes hídricas generando una especie de manto vegetal. Además sus raíces son blancas y finas y al mismo tiempo se trata de una planta subacuática que necesita de cierto nivel de inundación de agua dulce para desarrollarse.

Shelley, como proyecto académico, sienta las bases para un nuevo sistema de cultivo autónomo auto sustentable. Una forma de cultivar que no necesita de energía, que se sostiene en su relación con el medioambiente y que basa su funcionamiento en un consorcio sintético-natural, donde ambas partes se nutren de los beneficios de la otra.

\section{Conclusiones}

Lo natural nos presenta un inigualable repertorio de ideas a las que podemos recurrir cuando buscamos inspiración. La forma en que extrapolamos estas adaptaciones estará supeditada a nuestra capacidad de conectar ámbitos que parecen correlacionalmente distantes pero que comparten desafíos y oportunidades. En este sentido, podemos aprovechar esta integración disciplinar como un mecanismo de innovación que reconoce en el proceso de ensayo y error evolutivo un recurso analógico de inmenso valor al proceso creativo.

Las múltiples aproximaciones que han hecho de la naturaleza su fuente de inspiración, seguirán avanzando, acortando las brechas disciplinares y abriendo campos de investigación y desarrollo con un tremendo potencial. Es en este contexto en el que el desarrollo de una metodología de resolución creativa que aprovecha, no solo la inspiración individual, sino que se basa en los procesos evolutivos de cooperación e integración, nos abre oportunidades innegables de vinculación transdisciplinar.

Ha sido un proceso que requiere seguir refinando sus procedimientos y fases para acercar estas metodologías a otros campos del conocimiento. Avanzar en brindar mecanismos de resolución analógica será una manera de traspasar los límites impuestos por la tipología del problema a resolver.

La ideación basada en simbiosis surge y se ha establecido como un modelo de trabajo en proyectos de título con estudiantes de Diseño, en ejercicios asociados a proyectos de divulgación científica con los estudiantes del taller Lowtech y Ciencia UC de la Escuela de Diseño de la Pontificia Universidad Católica de Chile, y como un modelo que avanza hacia validaciones disciplinares integrando nuevos mecanismos de interacción interespecíficas positivas existentes en el mundo natural.

En la actualidad estamos en el desarrollo de una plataforma que caracterice las adaptaciones biológicas y permita conectar estas prestaciones en nuevas soluciones generadas desde la integración interespecies.

Somos simbiontes en un ecosistema simbiótico y, al observar en detalle, podemos reconocer que la cooperación es un fenómeno omnipresente y catalizador de la innovación evolutiva. 


\section{Bibliografía / Referencias}

Boden, M. A. (1996). Creativity. In Artificial intelligence (pp. 267-291). Academic Press.

Camere, S. \& Karana, E. (2018). Fabricating materials from living organisms: An emerging design practice. Journal of Cleaner Production, 186, 570-584.

Cordua, C. (1996). Wittgenstein: análogos del lenguaje. Areté, 8(2), 191-213.

de Bary, A. (1879). Die erscheinung der symbiose. Verlag von Karl J. Trubner, Strassburg.

Dumas, J. S. \& Redish, J. (1999). A practical guide to usability testing. Intellect books.

Durán, A. \& O’Ryan, L. (2019). Symbiogenesis-Based Design: a novel methodological approach to design based on cooperation and integration. The Design After, Cumulus Conference Proceedings Series Bogota 2019, 554-567.

Fayemi, P. E.; Maranzana, N.; Aoussat, A. \& Bersano, G., "Bio-inspired design characterisation and its links with problem solving tools," Proc. Int. Des. Conf. Des. 2014-Janua, D. Marjanović; M. Štorga; N. Pavković, and N. Bojčetić, Eds., 173-182, The Design Society, Glasgow, UK. (2014).

Fujii, H.; Yoshida, K. \& Sugimura, K. (2016). Research and development strategy in biological technologies: A patent data analysis of Japanese manufacturing firms. Sustainability, $8(4), 351$.

Helms, M.; Vattam, S. S. \& Goel, A. K., "Biologically inspired design: process and products," Des. Stud. 30(5), 606-622 (2009).

ISO 18458 (2015). “Biomimetics-Terminology, Concepts and Methodology,” ISO 2015.

Lenau, T. A.; Metze, A. L. \& Hesselberg, T. (2018). Paradigms for biologically inspired design. In Bioinspiration, Biomimetics, and Bioreplication VIII (Vol. 10593, p. 1059302). International Society for Optics and Photonics.

Margulis, L. \& Fester, R. (Eds.). (1991). Symbiosis as a source of evolutionary innovation: speciation and morphogenesis. Mit Press.

Margulis, L. \& Sagan, D. (2008). Acquiring genomes: A theory of the origin of species. Basic books.

Margulis, L. and Dorion, S. (1998). Microcosmos: Four Billion Years of Evolution from Our Microbial Ancestors. University of California Press.

Mironov, V.; Trusk, T.; Kasyanov, V.; Little, S.; Swaja, R. \& Markwald, R. (2009). Biofabrication: a 21st century manufacturing paradigm. Biofabrication, 1(2), 022001.

Risbud, A. S. \& Bartl, M. H. (2013). Engineered Biomimicry: Chapter 14. Solution-Based Techniques for Biomimetics and Bioreplication. Elsevier Inc. Chapters.

Rojas, D. and Durán, A.(2017). Shelley: Soft-robot sintético-natural acuático, energéticamente sustentable; oxigenador por movimiento y purificador por plantas. Tesis presentada a Escuela de Diseño UC.

Shu, L. H.; Ueda, K.; Chiu, I. \& Cheong, H. (2011). Biologically inspired design. CIRP Annals, 60(2), 673-693.

Vandevenne, D.; Verhaegen, P. A.; Dewulf, S. \& Duflou, J. R. (2011). A scalable approach for the integration of large knowledge repositories in the biologically-inspired design process. In DS 68-6: Proceedings of the 18th International Conference on Engineering Design (ICED 11), Impacting Society through Engineering Design, Vol. 6: Design Information and Knowledge, Lyngby/Copenhagen, Denmark, 15.-19.08. 2011 (pp. 210-219). 
Abstract: The origin of ideas depends in many cases on the analogue capacity assumed by the designer to extrapolate the qualities from the source of inspiration. When this inspiration comes from the natural, we recognize it as Biologically Inspired Design.

This article presents a summary of these approaches, together with the potential of Design Based on Symbiogenesis as a model that, in addition to recognizing the value of biological analogy, incorporates evolutionary dynamics associated with the origin of species through cooperation and integration through symbiosis.

Keywords: Biologically Inspired Design (BID) - Design Based on Symbiogenesis - Problem-driven BID - Solution-driven BID - symbiont.

Resumo: A origem das ideias depende, em muitos casos, da capacidade analógica assumida pelo designer de extrapolar as qualidades da fonte de inspiração. Quando essa inspiração vem do natural, nós o reconhecemos como um design inspirado na biologia.

Este artigo apresenta um resumo dessas abordagens, juntamente com o potencial do Design Based on Symbiogenesis como um modelo que, além de reconhecer o valor da analogia biológica, incorpora a dinâmica evolutiva associada à origem das espécies por meio da cooperação e integração, por meio da simbiose.

Palavras chave: Biologicamente Inspirado Design (BID) - Design Baseado em Symbiogenesis - Problem-driven BID - Solution-driven BID - symbiont.

[Las traducciones de los abstracts fueron supervisadas por el autor de cada artículo] 